Trauma Berufskrankh 2016 · [Suppl 1]: 18:S92-S95 DOI 10.1007/s10039-015-0054-0

Online publiziert: 23. Juni 2015

(c) Springer-Verlag Berlin Heidelberg 2015

CrossMark

\section{R. Martínez}

Abteilung BG Neurochirurgie und Neurotraumatologie, Klinik für Chirurgie, Berufsgenossenschaftliches

Universitätsklinikum Bergmannsheil Bochum, Ruhr-Universität Bochum, Bochum

\section{Neuromonitoring und operative Therapie beim schweren Schädel-Hirn-Trauma}

zung von Mediatorsubstanzen wie exzitatorischer Aminosäuren, freier Radikalen, Stickoxid oder Arachidonsäure und anderer inflammatorischer Mediatoren, die für die Entstehung des Sekundärschadens verantwortlich sind. Der Sekundärschaden kann wiederum durch das Auftreten weiterer Noxen, wie einer arteriellen Hypotension oder einer Hypoxie, verstärkt werden (• Abb. 2). Das therapeutische Management solcher Kaskaden ist das Ziel der Neuroprotektion [11, 14, 15].

\section{Management und Therapie des SHT}

In den letzten Jahren wird das derzeit bestehende Konzept des Monitorings beim schweren SHT kontrovers diskutiert. Frühere Studien internationaler Fachgesellschaften $[2,3,16,17]$ in den Jahren 1995 bis 2007 betonten den Stellenwert der Messung des intrakraniellen Drucks (ICP) und des zerebralen Perfusionsdrucks (CPP) im Rahmen des Monitorings. Im Verlauf setzte sich jedoch zunehmend das Konzept des sog. multimodalen Monitorings durch. Dieses umfasst neben der ICP- und CPP-Messung weitere Parameter und Hirnfunktionen, wie die zerebrale Oxygenierung (Sauerstoffpartialdruck, Oxymetrie im Bereich des Bulbus venae jugularis, „near-infrared spectroscopy“) und die zerebrale Perfusion („thermal diffusion flowmetry“, Xenon-Computertomographie, Positronenemissionstomographie, Einzelphotonenemissionscomputertomographie), die Elektrophysiologie (Elektroenzephalographie, somatosensibel evozierte Potenziale) sowie den Metabolismus des Gehirns mithilfe der Mikrodialyse ([9], • Abb. 3).

Im Jahre 2012 veröffentlichten Chesnut et al. [4] eine prospektive randomisierte SHT-Studie, in der kein signifikan-
Pathophysiologisch muss beim SHT zwischen einem primären und einem sekundären Hirnschaden unterschieden werden (- Abb. 1). Der Primärschaden ist die Läsion, die durch die traumatisch bedingte Einwirkung auf das Hirngewebe verursacht wird. Sie ist durch Therapiemaßnahmen nicht mehr zu beeinflussen. Die sekundäre Hirnschädigung tritt durch extra- oder intrakranielle Ursachen zusätzlich zum Primärschaden auf. Hierfür kommen sowohl systemische Komplikationen, aber auch im Hirngewebe ablaufende pathophysiologische Kaskaden infrage. Als häufige systemische Komplikationen sind eine Hypoxie oder eine arterielle Hypotension zu nennen. Bei den durch den Primärschaden ausgelösten Kaskaden hervorzuheben ist die Freiset-

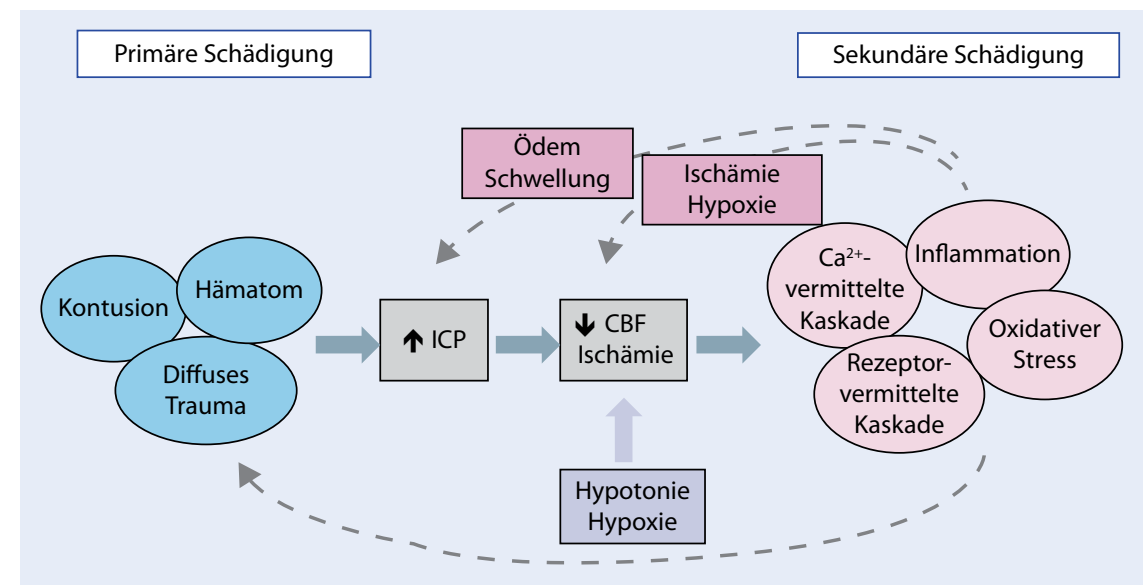

Abb. $1 \Delta$ Verlauf des Primärschadens beim schweren Schädel-Hirn-Trauma mit Erhöhung des intrakraniellen Drucks (ICP) und Reduktion des zerebralen Blutflusses (CBF) sowie die Interaktion intrazerebraler (pathophysiologische Kaskaden) und extrazerebraler systemischer Noxen (Hypotonie, Hypoxie), die zum Sekundärschaden führen 


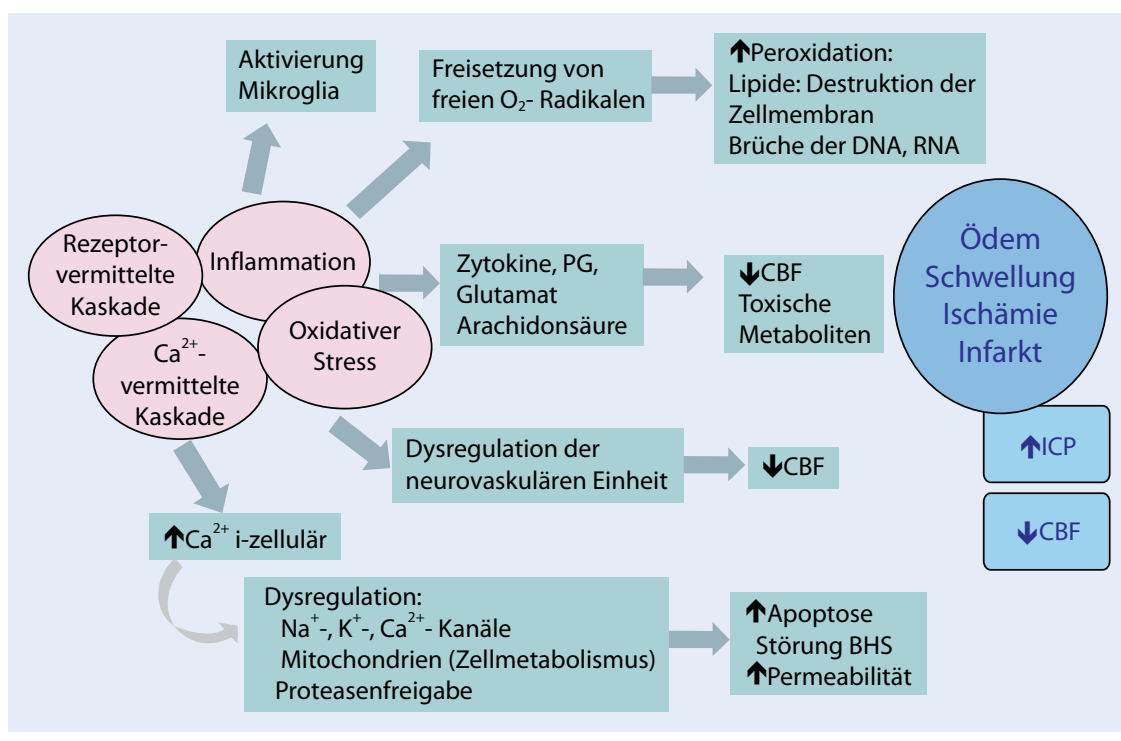

Abb. 2 \ Pathophysiologische Kaskaden nach Schädel-Hirn-Trauma, die zum Sekundärschaden führen. Unter anderem sind dies die Aktivierung der Mikroglia, die Freisetzung von freien $\mathrm{O}_{2}$-Radikalen, Zytokinen, Prostaglandinen (PG) und Arachidonsäure sowie die Dysregulation der sog. neurovaskulären Einheit und die $\mathrm{Ca}^{2+}$-vermittelte Dysregulation von Zellmembrankanälen und metabolischen Prozessen, die innerhalb der Mitochondrien stattfinden. BHS Blut-Hirn-Schranke, CBF zerebraler Blutfluss, ICP intrakranieller Druck, i-zellulär intrazellulär

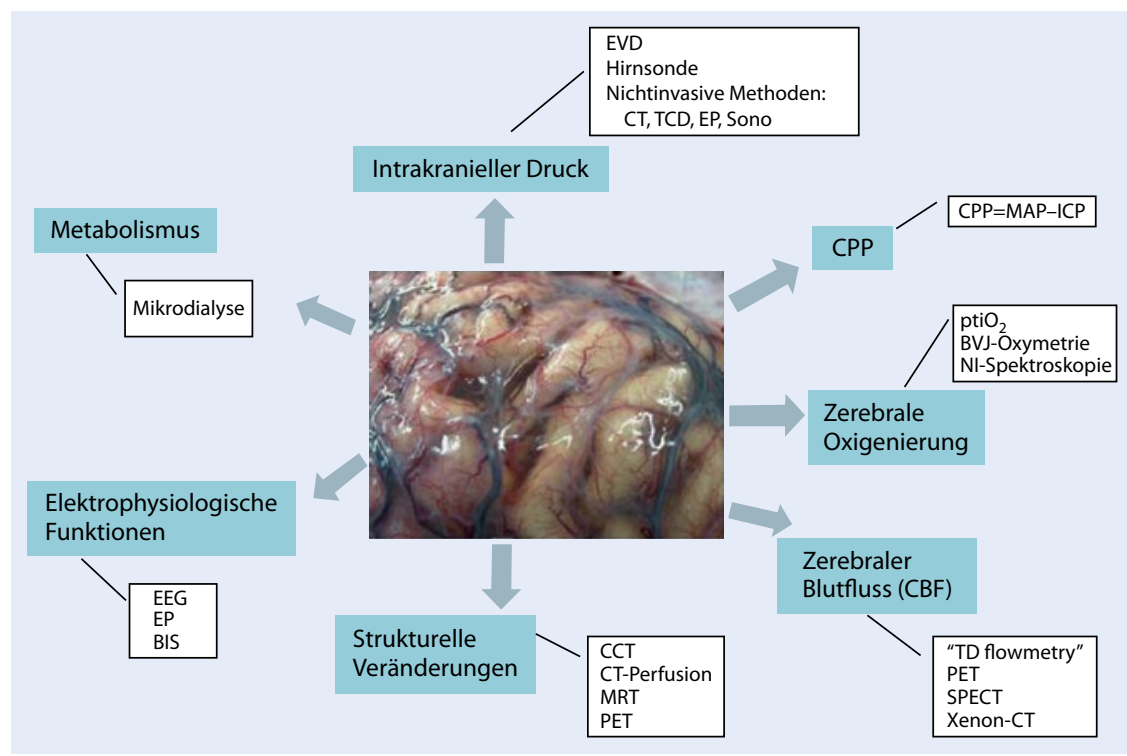

Abb. $3 \Delta$ Überblick über Hirnfunktionen und Parameter und ihr Monitoring. BIS bispektraler Index (Monitoring), BVJ Bulbus vena jugularis (Oxymetrie), CCT kraniale Computertomographie, CBF zerebraler Blutfluss, $C P P$ zerebraler Perfusionsdruck, EEG Elektroenzephalographie, EP evozierte Potentiale, EVD externe Ventrikeldrainage, ICP intrakranieller Druck, MAP mittlerer arerieller Blutdruck, MRT Mag-

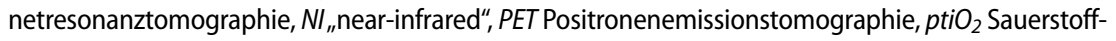
partialdruck, SPECT Einzelphotonenemmissions-CT, TCD transkranielle Dopplersonographie, $T D_{\text {", ther- }}$ mal diffusion"

ter Unterschied festgestellt werden konnte hinsichtlich des Outcomes von SHT-Patienten ohne ICP-Monitoring verglichen mit solchen Patienten, bei denen eine ICPMessung durchgeführt worden war. Diese Studie führte zu einer internationalen kontroversen Diskussion über das Neuromonitoring beim SHT.

Aufgrund von Einschränkungen im Studiendesign [5] und basierend auf weiteren nach 2012 erschienenen prospektiven Studien liegen nun Konsensuspapiere vor, die die Nutzung invasiver ICP-Messungen empfehlen. Hierbei wird das Vorhandensein eines pathologischen Befunds der kraniellen Computertomographie (CCT) in komatösen Patienten als Indikation zum invasiven ICP-Monitoring angegeben $[6,7,23]$. Darüber hinaus soll eine invasive ICP-Messung auch nach einer operativen Entfernung raumfordernder Hämatome erfolgen, wenn aufgrund des Volumens der Raumforderung oder des intraoperativen Befunds eine Reduktion der Analgosedation (und damit die Möglichkeit einer neurologischen Beurteilung des Patienten) zunächst nicht möglich ist.

Da der zerebrale Primärschaden und die mechanische Einwirkung auf das Hirngewebe nicht beinflussbar sind und die toxische Wirkung eines Sekundärschadens auf zellulärer Ebene derzeit durch effektive regenerative Maßnahmen nicht therapiert werden kann, steht die Prävention im Hinblick auf das Auftreten von Sekundärschäden in Vordergrund. Hierbei spielt das Neuromonitoring eine entscheidende Rolle. Auch sind Maßnahmen zur Diagnostik und Therapie systemischer Komplikationen, die zu Sekundärschäden führen können, wichtig. Zu nennen sind neben der respiratorischen und zirkulatorischen Stabilisierung des Patienten die Vermeidung von Hypoxie, Hypotension, Hyper- und Hypokapnie, Hyperthermie, Hyper- und Hypoglykämie sowie einer Hypernatriämie.

Neurochirurgische Maßnahmen kommen erst dann infrage, wenn alle durchgeführten konservativen Verfahren (Oberkörperhochlage von $30^{\circ}$, Optimierung der Oxygenierung, Ventilation, Körpertemperatur und metabolische Parameter, tiefe Analgosedation bzw. Relaxation und antiosmotische Therapie mit Mannitol oder hyperosmolaren Lösungen) nicht in der Lage sind, eine intrakranielle Hypertension zu kontrollieren. Hierbei stehen zunächst die Liquordrainage (über eine externe Ventrikeldrainage), die Entfernung von Raumforderungen (z. B. einer Kontusionsblutung) oder die dekompressive Hemikraniektomie zur Verfügung [1, 2, 19].

Bei primären intrakraniellen Raumforderungen als Folge des mechanischen Primärschadens, etwa bei einem epiduralen Hämatom, einem akuten Subduralhäma- 
tom oder bei Kontusionblutungen, sind operative neurochirurgische Maßnahmen primär $\mathrm{ab}$ einem bestimmten $\mathrm{Vo}$ lumen der intrakraniellen Läsionen oder bei klinischen Hinweisen auf eine intrakranielle Hypertension erforderlich ([8, 10, 13]; • Abb. 4).

\section{Schlussfolgerungen}

Dank der Verbesserung im SHT-Management, in der intensivmedizinischen Behandlung und in der operativen Therapie in spezialisierten Traumazentren ist die Mortalität bei Patienten mit einem schweren SHT in den letzten Jahren kontinuierlich gesunken. Die rasante technische Entwicklung im neurochirurgischen Operationssaal mit Hochleistungsmikroskopen und dem intraoperativen Ultraschall oder den intraoperativen Dopplersonographiegeräten hat entscheidend hierzu beigetragen. So lag die Mortalitätsrate in Deutschland 1972 bei 27,2 pro 100.000 Einwohner und 2000 bei 9 Sterbefällen pro 100.000 Einwohner [22]. Auch nach den Ergebnissen der National Data Trauma Bank (www.facs.org/trauma/ntdb/index.html), dem IMPACT (www.tbi-impact.org), den WHO-Daten [26] und prospektiven bzw. retrospektiven Metanalysen fiel die Mortalität bei Patienten nach einem SHT von $33 \%$ auf $11 \%$ [12, 20, 24].

Ebenso sind für die Nachsorge der Patienten Verbesserungen in Bezug auf eine standardisierte medikamentöse Therapie und auf Rehabilitationsmaßnahmen der neurokognitiven, psychologischen und systemischen Spätfolgen zu verzeichnen [25].

\section{Fazit}

- Das SHT stellt eine große gesundheitliche und soziale Herausforderung dar. Trotz einer großen Anzahl vielversprechender Ergebnisse aus experimentellen Studien ist es bis heute nicht gelungen, eine neuroprotektive Therapie in der Klinik zu etablieren.

- Fortschritte in der Prävention und eine Optimierung des Managements des schweres SHT spielen eine groBe Rolle.

Trauma Berufskrankh 2016 • [Suppl 1]: 18:S92-S95 DOI 10.1007/s10039-015-0054-0

(c) Springer-Verlag Berlin Heidelberg 2015

\section{R. Martínez}

\section{Neuromonitoring und operative Therapie beim schweren Schädel-Hirn-Trauma}

\section{Zusammenfassung}

Hintergrund. Das Schädel-Hirn-Trauma (SHT) ist in industrialisierten Ländern die wichtigste Ursache für die Mortalität und Morbidität im Lebensalter von 20 bis 40 Jahren. Pathophysiologisch wird zwischen dem Primärschaden (Läsion des Gehirns nach einer mechanischen Einwirkung) und dem Sekundärschaden (Schädigung des Gehirns nach sekundär aufgetretenen Noxen, hauptsächlich Hirnödem und Hirnschwellung) unterschieden. Das Management von SHT-Patienten zielt darauf ab, sekundäre Komplikationen zu vermeiden bzw. zu behandeln. Management und Therapie. Trotz einer großen Anzahl vielversprechender experimenteller Studien ist es bis heute nicht gelungen, eine neuroregenerative Therapie in der Klinik zu etablieren. Daher ist die Rolle des Neuromonitorings zur Prävention des Sekundärschadens nach einem SHT entscheidend. Unterschiedliche Methoden zur Analyse verschiedener Hirnparameter stehen derzeit zur
Verfügung. Im klinischen Alltag haben sich die Messung des intrakraniellen Drucks (ICP), der zerebralen Oxygenierung mittels Sauerstoffpartialdrucks $\left(\mathrm{ptiO}_{2}\right)$, des zerebralen Perfusionsdrucks (CPP) sowie Untersuchungen zu strukturellen intrakraniellen Veränderungen (neuroradiologische Untersuchungen) und zu Hirnfunktionen (elektrophysiologische Untersuchungen) als Routineverfahren etabliert. Die chirurgische Entfernung von Raumforderungen wie Epi- oder Subduralhämatome und Kontusionsblutungen bzw. die dekompressive Hemikraniektomie sind neurochirurgische Maßnahmen, die initial oder während der akuten Phase eines schweren SHT zur Verfügung stehen.

Schlüsselwörter

Schädelhirntrauma · Neuromonitoring · Neurochirurgie · Präventivmaßnahmen . Regeneration

\section{Neuromonitoring and surgical therapy for severe traumatic brain injury}

\section{Abstract}

Background. Traumatic brain injury (TBI) is a leading cause of morbidity, disability and mortality worldwide. From a pathophysiological point of view, a differentiation must be made between primary injury due to mechanical injury to the brain and secondary injury due to the occurrence of secondary noxious events e.g. brain edema and swelling. The major goals of TBI management are prevention and treatment of secondary injuries. Management and therapy. Many experimental studies have been carried out to find effective neuroregenerative strategies but none of them could successfully be translated from benchmarking to the patient bedside. Thus, the role of neuromonitoring is of decisive importance to avoid secondary injuries in TBI patients. Management of TBI in- cludes monitoring of intracranial pressure (ICP), cerebral tissue oxygenation by oxygen partial pressure $\left(\mathrm{ptiO}_{2}\right)$, cerebral perfusion pressure (CPP) as well as neuroradiological and electrophysiological assessments. The surgical removal of TBI-associated lesions with mass effects, such as epidural and subdural hematomas and contusion bleeding, and decompressive craniectomy are well-established neurosurgical procedures which are performed initially or during the critical phase of severe TBI.

Keywords

Traumatic brain injuries . Neurophysiological monitoring · Neurosurgery · Preventive measures $\cdot$ Regeneration

\section{- Nach der aktuellen wissenschaftli- chen Datenlage ist die ICP/CPP-ba- sierte Therapie des schweren SHT bei komatösen Patienten mit einem pa- thologischen CCT zu empfehlen.}
- Bei bestehenden Indikationen sind neurochirurgische Maßnahmen not- wendig und führen in kontrollierten Studien zu einer signifikanten Verbes- serung des Outcome.


Indikation:

Kontusionsblutung

Epidurales Hämatom

Akutes subdurales Hämatom

Blutung hintere Schädelgrube
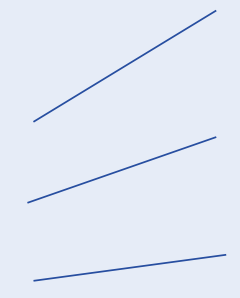

Volumen $>50 \mathrm{ml}$

ICP $>20 \mathrm{mmHg}$, $>30 \mathrm{~mm}$

Neurologische Defizite

Volumen $>30 \mathrm{ml}$

(unabhängig vom ICP)

\section{Dicke $>10 \mathrm{~mm}$}

MLV $>5 \mathrm{~mm}$

ICP > $20 \mathrm{mmHg},>30 \mathrm{~min}$

Anisokorie

\section{Ventrikel Kompression}

Blutung $>30 \mathrm{~mm}$

Neurologische Defizite

"Verlust" der basalen Zisternen

Abb. $4 \Delta$ Operationsindikationen bei SHT-assoziierten intrakraniellen Pathologien mit raumfordernder Wirkung. ICP intrakranieller Druck, MLV Mittellinienverlagerung

\section{Korrespondenzadresse}

\section{R. Martínez MD, PhD}

Abteilung BG Neurochirurgie und Neurotraumatologie, Klinik für Chirurgie Berufsgenossenschaftliches Universitätsklinikum Bergmannsheil Bochum Ruhr-Universität Bochum Bürkle-de-la-Camp-Platz 1, 44789 Bochum ramon.martinez@bergmannsheil.de

\section{Einhaltung ethischer Richtlinien}

Interessenkonflikt. R. Martínez gibt an, dass kein Interessenkonflikt besteht.

Dieser Beitrag beinhaltet keine Studien an Menschen oder Tieren.

The supplement containing this article is not sponsored by industry

\section{Literatur}

1. Bor-Seng-Shu E, Figueiredo EG, Amorim RL, Teixeira MJ, Valbuza JS, de Oliveira MM, Panerai RB (2012) Decompressive craniectomy: a meta-analysis of influences on intracranial pressure and cerebral perfusion pressure in the treatment of traumatic brain injury. J Neurosurg 117(3):589-596

2. (2007) Brain Trauma Foundation. The American Association of Neurological Surgeons. The Joint Section on Neurotrauma and Critical Care. J Neurotrauma 24(Suppl 1):1-106

3. Bullock R, Chesnut RM, Clifton G, Ghajar J, Marion DW, Narayan RK, Newell DW, Pitts LH, Rosner MJ, Wilberger JE (1996) Guidelines for the management of severe head injury. J Neurotrauma 13:639-734

4. Chesnut RM, Temkin N, Carney N, Dikmen S, Rondina C, Videtta W, Petroni G, Lujan S, Pridgeon J, Barber J, Machamer J, Chaddock K, Celix JM, Cherner $M$, Hendrix T, Global Neurotrauma Research Group (2012) A trial of intracranial-pressure monitoring in traumatic brain injury. N Engl J Med 367(26):2471-2481
5. Chesnut R, Videtta W, Vespa P, Le Roux P (2014) Intracranial pressure monitoring: fundamental considerations and rationale for monitoring. Participants in the International Multidisciplinary Consensus Conference on Multimodality Monitoring. Neurocrit Care 21(Suppl 2):64-84

6. Dawes A, Sacks G, Cryer H, Gruen P, Preston C, Gorospe $D$, Cohen $M$, McArthur D, Russell M, Maggard-Gibbons M, Ko C (2015) Intracranial pressure monitoring and inpatient mortality in severe traumatic brain injury: a propensity score matched analysis. JTrauma Acute Care Surg 78(3):492-502

7. Farahvar A, Gerber LM, Chiu YL, Carney N, Härtl R, Ghajar J (2012) Increased mortality in patients with severe traumatic brain injury treated without intracranial pressure monitoring. J Neurosurg 117(4):729-734

8. Feinberg M, Mai JC, Ecklund J (2015) Neurosurgical management in traumatic brain injury. Semin Neurol 35:50-56

9. Feyen BF, Sener S, Jorens PG, Menovsky T, Maas AI (2012) Neuromonitoring in traumatic brain injury. Minerva Anestesiol 78(8):949-958

10. Flynn-O’Brien KT1, Fawcett VJ, Nixon ZA, Rivara FP, Davidson GH, Chesnut RM, Ellenbogen RG, Vavilala MS, Bulger EM, Maier RV, Arbabi S (2015) Temporal trends in surgical intervention for severe traumatic brain injury caused by extra-axial hemorrhage, 1995 to 2012. Neurosurgery 76(4):451-460

11. Garry PS, Ezra M, Rowland MJ, Westbrook J, Pattinson KT (2015) The role of the nitric oxide pathway in brain injury and its treatment-from bench to bedside. Exp Neurol 263:235-243

12. Gerber LM1, Chiu YL, Carney N, Härtl R, Ghajar J (2013) Marked reduction in mortality in patients with severe traumatic brain injury. J Neurosurg 119(6):1583-1590

13. Grandhi R, Bonfield CM, Newman WC, Okonkwo DO (2014) Surgical management of traumatic brain injury: a review of guidelines, pathophysiology, neurophysiology, outcomes, and controversies. J Neurosurg Sci 58(4):249-259

14. Harish G1, Mahadevan A, Pruthi N, Sreenivasamurthy SK, Puttamallesh VN, Keshava Prasad TS, Shankar SK, Srinivas Bharath MM (2015) Characterization of traumatic brain injury in human brains reveals distinct cellular and molecular changes in contusion and pericontusion. J Neurochem. doi:10.1111/jnc.13082
15. Kochanek PM, Jackson TC, Ferguson NM, Carlson SW, Simon DW, Brockman EC, Ji J, Bayır H, Poloyac SM, Wagner AK, Kline AE, Empey PE, Clark RS, Jackson EK, Dixon CE (2015) Emerging therapies in traumatic brain injury. Semin Neurol 35(1):83-100

16. Lee JC, Rittenhouse K, Bupp K, Gross B, Rogers A, Rogers FB, Horst M, Estrella L, Thurmond J (2015) An analysis of Brain Trauma Foundation traumatic brain injury guideline compliance and patient outcome. Injury 46(5):854-858

17. Maas Al, Dearden M, Servadei F, Stocchetti N, Unterberg A (2000) Current recommendations for neurotrauma. Curr Opin Crit Care 6(4):281-292

18. Rusnak M (2013) Traumatic brain injury: giving voice to a silent epidemic. Nat Rev Neurol 9(4):186187

19. Sahuquillo J, Arikan F (2006) Decompressive craniectomy for the treatment of refractory high intracranial pressure in traumatic brain injury. Cochrane Database Syst Rev 25(1):CD003983

20. Scholten A, Haagsma J, Panneman M, van Beeck E, Polinder S2 (2015) Traumatic brain injury in the Netherlands: incidence, costs and disability-adjusted life years. PLOS One 9(10):e110905. doi:10.1371

21. Spitz G, Downing MG, McKenzie D, Ponsford JL (2015) Mortality following traumatic brain injury inpatient rehabilitation. J Neurotrauma [Epub ahead of print]

22. Steudel WI, Cortbus F, Schwerdtfeger K (2005) Epidemiology and prevention of fatal head injuries in Germany - trends and the impact of the reunification. Acta Neurochir (Wien) 147(3):231-242

23. Stocchetti N, Picetti E, Berardino M, Buki A, Chesnut RM, Fountas KN, Horn P, Hutchinson PJ, laccarino C, Kolias AG, Koskinen LO, Latronico N, Maas Al, Payen JF, Rosenthal G, Sahuquillo J, Signoretti S, Soustiel JF, Servadei F (2014) Clinical applications of intracranial pressure monitoring in traumatic brain injury: report of the Milan consensus conference. Acta Neurochir (Wien) 156(8):1615-1622

24. Tagliaferri F, Compagnone C, Korsic M, Servadei F, Kraus J (2006) A systematic review of brain injury epidemiology in Europe. Acta Neurochir (Wien) 148:255-268

25. Tate R, Kennedy M, Ponsford J, Douglas J, Velikonja D, Bayley M, Stergiou-Kita M (2014) INCOG recommendations for management of cognition following traumatic brain injury, part III: executive function and self-awareness. J Head Trauma Rehabil 29(4):338-352

26. World Health Organization (2006) Neurological disorders: public health challenges. WHO, Geneva. www.who.int/mental health/neurology/neurological_disorders_report. ISBN 9241563362 (NLM classification: WL 140), ISBN 978924156336 9. www.who.int 